\title{
Paclitaxel inhibits proliferation and invasion and promotes apoptosis of breast cancer cells by blocking activation of the PI3K/AKT signaling pathway
}

\author{
${ }^{*}$ Gang $\mathrm{Li}^{1, A-D}$, ${ }^{*}$ Dongxin $\mathrm{Xu}^{2, A-D}$, Jinju Sun ${ }^{3, C, D}$, Shiyun Zha0 ${ }^{4, A, C, D}$, Dan Zheng ${ }^{5, E, F}$ \\ ${ }^{1}$ First Department of Oncology Surgery, Hangzhou Cancer Hospital, China \\ ${ }^{2}$ Fourth Department of Internal Medicine, Zibo City Traditional Chinese Medicine Hospital, China \\ ${ }^{3}$ Department of Pharmacy, The Second People's Hospital of Liaocheng, China \\ ${ }^{4}$ Disinfection Supply Center, Maternal and Child Health Care of Zaozhuang, China \\ ${ }^{5}$ Department of Pharmacy, Hangzhou Cancer Hospital, China \\ A - research concept and design; $\mathrm{B}$ - collection and/or assembly of data; $\mathrm{C}$ - data analysis and interpretation; \\ $\mathrm{D}$ - writing the article; $\mathrm{E}$ - critical revision of the article; $\mathrm{F}$ - final approval of the article
}

\author{
Address for correspondence \\ Dan Zheng \\ E-mail: zhengdanqazx12@163.com \\ Funding sources \\ None declared \\ Conflict of interest \\ None declared \\ *Gang Li and Dongxin Xu contributed equally \\ to this work.
}

Received on September 20, 2019

Reviewed on June 13, 2020

Accepted on September 20, 2020

Cite as

Li G, Xu D, Sun J, Zhao S, Zheng D. Paclitaxel inhibits proliferation and invasion and promotes apoptosis of breast cancer cells by blocking activation of the PI3K/AKT signaling pathway. Adv Clin Exp Med. 2020;29(11):1337-1345. doi:10.17219/acem/127681

DOI

10.17219/acem/127681

Copyright

Copyright by Author(s)

This is an article distributed under the terms of the

Creative Commons Attribution 3.0 Unported (CC BY 3.0)

(https://creativecommons.org/licenses/by/3.0/)

\section{Abstract}

Background. Breast cancer has the highest incidence and mortality among all cancers in women. Paclitaxel (PTX) has a notable therapeutic effect on cancer in clinical practice.

Objectives. To explore the effect and mechanism of PTX on the proliferation, apoptosis and invasiveness of breast cancer cells.

Material and methods. MCF-7 cells were treated with PTX (0 $\mu \mathrm{M}, 0.01 \mu \mathrm{M}, 0.1 \mu \mathrm{M}, 1 \mu \mathrm{M})$ for $48 \mathrm{~h}$. Cell viability was detected using MTT assay and lactate dehydrogenase (LDH) assay; the cell proliferation rate was detected using 5-ethynyl-2'-deoxyuridine (EdU) assay to screen the most effective concentration of PTX. MCF-7 cells were then divided into 5 groups: control group, PTX group, oe-PI3K group, NC-PI3K group, and oe-PI3K+PTX group. Cell apoptosis and cell cycles were detected with flow cytometry; cell invasion was determined using a transwell assay; western blot and quantitative reverse-transcription polymerase chain reaction (qRT-PCR) were used to measure the mRNA and protein expression level of cleaved caspase-3, Bax, BCl-2, matrix metalloproteinase 9 (MMP-9), vascular endothelial growth factor (VEGF), p-AKT (Thr308), and p-AKT (Ser473).

Results. Paclitaxel inhibited cell viability and proliferation in a dose-dependent manner. In the PTX group, the apoptosis rate, the number of cells arrested in the G2/M phase and the expression levels of Cleaved caspase-3 and Bax were increased, but the number of invasive cells and the expression levels of BCl-2, MMP-9, vascular endothelial growth factor (VEGF), p-AKT (Thr308), and p-AKT (Ser473) were decreased. However, PI3K upregulation can reverse the effects of PTX.

Conclusions. Paclitaxel could inhibit MCF-7 cell proliferation and invasion, and promote MCF-7 cell apoptosis by downregulating the expression of p-AKT (Thr308) and p-AKT (Ser473) in the PIJK/AKT signaling pathway.

Key words: apoptosis, breast cancer, proliferation, paclitaxel, PI3K/AKt signaling pathway 


\section{Introduction}

Breast cancer is the most common gynecological cancer, with the highest mortality rate. More than 1.5 million women are diagnosed with breast cancer each year worldwide, and this number is rising. ${ }^{1,2}$ The common classification of breast cancer is based on the expression of estrogen receptor (ER), progesterone receptor (PR) and human epidermal growth factor receptor (HER2). ${ }^{3}$ There are many factors affecting the prevalence of breast cancer, including family history, age, smoking and drinking, early amenorrhea, delayed menarche, obesity, and high-fat diet; oral contraceptives or other exogenous estrogens can also increase the incidence of breast cancer. ${ }^{4-6}$ It is a highly heterogeneous disease - its clinical characteristics and biological behavior vary widely among patients; therefore, survival also varies from person to person. ${ }^{7}$ At present, radiotherapy and chemotherapy are the main methods of inhibiting the growth of breast cancer in order to prolong survival. However, the outcomes of current treatment regimens are often unsatisfactory due to recurrences of the disease caused by chemotherapy drug resistance. ${ }^{8-10}$ Therefore, fully understanding the mechanism of action of chemotherapy drugs may help improve the therapeutic outcome of breast cancer.

Paclitaxel (PTX) is a common chemotherapy drug that is widely used in clinical practice due to its remarkable therapeutic effect. ${ }^{11}$ It can hinder cell mitosis by maintaining tubulin stability. ${ }^{12}$ Paclitaxel can be used alone or in combination with other drugs to treat a variety of malignancies, including cervical cancer, head and neck cancers, and lung cancer. ${ }^{13-17}$ Paclitaxel is also the first-line chemotherapy for breast cancer. ${ }^{18}$

PI3K/AKT is one of the most frequently activated signaling pathways in cancer, which regulates cell proliferation and invasion by targeting multiple proteins. ${ }^{19}$ It has been reported that activation of the PI3K/AKT pathway can promote growth, invasion and metastasis of breast cancer cells. ${ }^{20}$

However, the relationship between PTX and the PI3K/AKT signaling pathway, and the mechanism of their interaction in the treatment of breast cancer have not been elucidated. Therefore, this study explored the relationship between PTX and the PI3K/AKT signaling pathway, with the hope of providing more insights for improving the therapeutic outcome of PTX in breast cancer patients.

\section{Material and methods}

\section{Cell culture and transfection}

Human breast cancer cell lines MCF-7, BT-549, MDAMB-231, and T47D, and immortalized human mammary epithelial cell line MCF-10A were purchased from the Shanghai Cell Bank of the Chinese Academy of Sciences (Beijing, China). The cells were cultured in minimal essential medium (MEM) containing 10\% fetal bovine serum (FBS) and $100 \mathrm{IU} / \mathrm{mL}$ penicillin in $5 \% \mathrm{CO}_{2}$ at $37^{\circ} \mathrm{C}$. The PI3K overexpression plasmid pcDNA3.1-PI3K and the negative control plasmid pcDNA3.1-NC were purchased from Vigene Biosciences Inc. (Jinan, China). Transfection was performed using a lipofectamin 2000 kit (Invitrogen, Carlsbad, USA). Briefly, $250 \mu \mathrm{L}$ of Opti-MEM (Gibco, Thermo Fisher Scientific, Waltham, USA) was used to dilute $4 \mu \mathrm{g}$ of the desired plasmid and $10 \mu \mathrm{L}$ of Lipofectamin 2000. The plasmid and Lipofectamin 2000 were allowed to stand at room temperature for $5 \mathrm{~min}$ before being gently mixed together. The mixture was allowed to stand for 20 min and then was added dropwise to the MCF-7 cell culture plate. The plate was gently shaken and put in an incubator for $6 \mathrm{~h}$ before the medium was changed. Cells were harvested at $48 \mathrm{~h}$ after transfection for later use.

\section{MTT assay}

When the MCF-7cells reached 80-90\% confluency, cells were digested and seeded into 96 -well plates. The cells were treated with gradient concentrations of PTX for $48 \mathrm{~h}$. After the medium was gently removed, $100 \mu \mathrm{L}$ medium containing 10\% MTT solution (Solarbio Science \& Technology Inc., Beijing, China) was added. After $4 \mathrm{~h}$, the supernatant was discarded; $110 \mu \mathrm{L}$ of dimethyl sulfoxide (DMSO) was then added to dissolve the crystal precipitate, and the absorbance value (optical density - OD) of each well at a wavelength of $490 \mathrm{~nm}$ was obtained using a microplate reader. Each group had 3 duplicate wells.

\section{Lactate dehydrogenase cytotoxicity assay}

The cell treatment was as described for the MTT assay (above). Cell culture medium was used as a blank control. Untreated MCF-7 cells were used as a spontaneous release control. Normal MCF-7 cells treated with Triton X-100 (Sigma-Aldrich, St. Louis, USA) were used as a maximum release control. In accordance with the instructions of the lactate dehydrogenase (LDH) cytotoxicity kit (Beyotime Biotechnology, Shanghai, China), $120 \mu \mathrm{L}$ of supernatant from each well was collected and transferred to a new 96-well plate. Then $60 \mu \mathrm{L}$ of LDH working reaction mixture was added to each well and incubated in the dark at room temperature for $30 \mathrm{~min}$. The absorbance of each well was measured at a wavelength of $490 \mathrm{~nm}$. Each sample had 3 replicate wells. For each test sample the cytotoxicity $(\%)=($ experimental value - spontaneous release $) /($ maximum release - spontaneous release) $\times 100 \%$.

\section{EdU cell proliferation assay}

The $\times 2$ EdU working solution was made by diluting $10 \mu \mathrm{M}$ of EdU (Beyotime) with MEM medium (1:500) and 
warmed to $37^{\circ} \mathrm{C}$. An equal volume of $\times 2$ EdU working solution was added to each well and the plate was incubated at room temperature for $2 \mathrm{~h}$, washed with PBS and fixed with $4 \%$ paraformaldehyde for $15 \mathrm{~min}$. The plates were then washed twice with PBS containing $3 \%$ bovine serum albumin (BSA) to remove the permeabilization solution. Click-i ${ }^{\circledR}$ cell buffer additive solution (Thermo Fisher Scientific) was added and each plate was incubated at room temperature for $30 \mathrm{~min}$ in the dark. Hoechst 33342 reaction solution was added, and the plates were incubated at room temperature for $10 \mathrm{~min}$ in the dark. The plates were washed 3 times before being observed under CKX53 inverted fluorescence microscope (Olympus, Tokyo, Japan). The red fluorescence was counted as proliferating cells, and the blue fluorescence showed nuclei. We chose 3 random $\times 200$ fields to count the proliferating cells with nuclei. The cell proliferation rate $=$ the number of proliferating cells/total cell number $\times 100 \%$.

\section{Flow cytometry}

\section{Cell apoptosis}

A cell apoptosis detection kit was purchased from Beyotime. Cells in each group were harvested and resuspended with PBS. A cell suspension containing $10^{4}$ cells was centrifuged and resuspended using working buffer. Then a staining solution was made by mixing Annexin V-FITC (Beyotime) and propidium iodide (PI) at a ratio of 1:2. The cells were mixed with the staining solution and incubated at room temperature for $20 \mathrm{~min}$. Cell apoptosis was measured in a flow cytometer; the experiment was repeated 3 times.

\section{Cell cycle}

A cell cycle detection kit was purchased from Beyotime. Cells were harvested, fixed with $1 \mathrm{~mL}$ of pre-cooled $75 \%$ ethanol $\left(-20^{\circ} \mathrm{C}\right)$ and stored overnight at $4^{\circ} \mathrm{C}$. The PI staining solution, staining buffer and Rnase A were made according to the instruction manual. The cells were washed twice with pre-cooled PBS, resuspended with PI staining solution and incubated at $37^{\circ} \mathrm{C}$ for $30 \mathrm{~min}$. The cell cycle was then detected using flow cytometry in the dark at $4^{\circ} \mathrm{C}$. The experiment was repeated 3 times.

\section{Transwell invasion assay}

Cells were starved for $12 \mathrm{~h}$ before the invasion assay. A 24-well plate with $8 \mu \mathrm{m}$ Transwell inserts (Corning Inc., Corning, USA) was used in this experiment; each group had 3 replicate inserts. The upper surface of each insert was coated with $50 \mu \mathrm{L}$ of Matrigel (Sigma-Aldrich) and airdried at $4^{\circ} \mathrm{C}$. We added $200 \mu \mathrm{L}$ of cell suspension to the upper chamber at a density of $1 \times 10^{5}$ cells $/ \mathrm{mL}$, and $500 \mu \mathrm{L}$ of Dulbecco's modified Eagle's medium (DMEM) medium containing $10 \%$ bovine serum to the lower chamber. After $48 \mathrm{~h}$, the inserts were taken out and the cells on the inserts were fixed with $4 \%$ paraformaldehyde for $30 \mathrm{~min}$. Then the insert was placed in $0.2 \%$ Triton X-100 for 15 min and stained with $0.05 \%$ gentian violet for $5 \mathrm{~min}$. We selected 5 random fields to count the number of transmembrane cells under an inverted microscope. The assay was repeated 3 times.

\section{Western blot}

Cells were harvested and resuspended with RIPA lysis buffer (Beyotime) containing phenylmethylsulfonyl fluoride (PMSF) and protein phosphatase inhibitors. The mixture was incubated on ice for $30 \mathrm{~min}$, and then centrifuged at $12,000 \mathrm{rpm}$ for $10 \mathrm{~min}$ at $4^{\circ} \mathrm{C}$. The supernatant was collected as total cell protein. The protein concentration was determined using the BCA Protein Quantitation Kit (Thermo Fisher Scientific). A total of $20 \mu \mathrm{g}$ of the protein in each sample was subjected to SDS-PAGE, and then the protein was wet transferred to a nitrocellulose (NC) membrane. The membrane was blocked with 5\% BSA for 90 min and incubated with rabbit primary antibodies, including 1:1000 anti-AKT (Cell Signalling Technology (CST) Inc., Danvers, USA), 1:1000 anti-p-AKT (Ser473) (CST), 1:1000 anti-p-AKT(Thr308) (CST), 1:1000 antimatrix metalloproteinase 9 (anti-MMP-9) (Proteintech Group Inc., Rosemont, USA), 1:1000 anti-vascular endothelial growth factor (anti-VEGF) (Proteintech Group Inc.), 1:1000 anti-cleaved caspase-3 (CST), 1:1000 Bax (CST), 1:10,000 anti- glyceraldehyde-3-phosphate dehydrogenase (anti-GAPDH) (Abcam, Cambridge, UK), and 1:2000 mouse primary antibody anti-Bcl- 2 (CST) at $4^{\circ} \mathrm{C}$ overnight. The membrane was rinsed 3 times with Tris-buffered saline with Tween 20 (TBST) for 10 min each time. Then the membrane was incubated with horseradish peroxidase (HRP)-conjugated goat anti-rabbit or goat anti-mouse antibody (1:10,000; Jackson Immunoresearch Laboratories Inc., West Grove, USA) at room temperature for $90 \mathrm{~min}$. The membrane was rinsed 3 times with TBST for 15 min each time, then developed using ECL solution and photographed using a SmartView Pro 2000 imager system (Major Science, Saratoga, USA). Relative protein expression was calculated using Image J software (National Institutes of Health (NIH), Bethesda, USA) with GAPDH as an internal reference. Relative protein expression = the grey value of each sample/the grey value of the internal reference.

\section{qRT-PCR}

Total RNAs were extracted from cells using Trizol (Invitrogen) and quantified using Nanodrop 2000 (Thermo Fisher Scientific). A PrimeScript ${ }^{\mathrm{TM}} \mathrm{RT}$ reagent kit with gDNA Eraser (TaKaRa Bio Inc., Kusatsu, Japan) was used for reverse transcription following the manufacturer's instructions. The reaction mixture was prepared using 
Table 1. qRT-PCR primer sequences

\begin{tabular}{l|c|c}
\multicolumn{1}{c|}{ Gene } & Forward primer $\left(5^{\prime}-3^{\prime}\right)$ & Reverse primer $\left(5^{\prime}-3^{\prime}\right)$ \\
\hline MMP-9 & AGACACCTCTGCCCTCACCATGAGC & TCTGTGGAGACGGGAGTGGTACTCG \\
\hline VEGF & CGGTGCTGGAATTGATA & GGCGGTGTCTGTCTGTCT \\
GAPDH & GGGTGTGAACCATGAGAAGTATG & GATGGCATGGACTGTGGTCAT \\
\hline
\end{tabular}

VEGF - vascular endothelial growth factor; MMP-9 - matrix metallopeptidase 9; GAPDH - glyceraldehyde-3-phosphate dehydrogenase.

a SYBR ${ }^{\circledR}$ Premix Ex Taq ${ }^{\mathrm{TM}}$ kit (TaKaRa Bio Inc.), and quantitative reverse-transcription polymerase chain reaction (qRT-PCR) was performed using an ABI7500 PCR system (Thermo Fisher Scientific). The reaction condition was as follows: pre-denaturation at $95^{\circ} \mathrm{C}$ for $10 \mathrm{~min}$, denaturation at $95^{\circ} \mathrm{C}$ for $15 \mathrm{~s}$ and annealing at $60^{\circ} \mathrm{C}$ for $30 \mathrm{~s}$; this cycle was repeated 40 times followed by extension at $72^{\circ} \mathrm{C}$ for $1 \mathrm{~min}$. Relative expression was calculated using the formula:

$$
\Delta \Delta \mathrm{Ct}=\left(\mathrm{Ct}_{\text {target gene }}-\mathrm{Ct}_{\mathrm{GAPDH}}\right)-\left(\mathrm{Ct}_{\text {control group }}-\mathrm{Ct}\right.
$$

with GAPDH as the internal reference. All the primers (Genepharma, Shanghai, China) are listed in Table 1.

\section{Statistical analysis}

All data was analyzed with SPSS v. 21.0 software (IBM Corp., Armonk, USA). Quantitative values were expressed as mean \pm standard deviation (SD). A one-way analysis of variance (ANOVA) followed by the Bonferroni post hoc test was used to compare the differences between groups. A p-value less than 0.05 was considered statistically significant.

\section{Results}

Expression of the PI3K/AKT signaling pathway-related protein p-AKT in breast cancer cell lines

The expression of PI3K/AKT signaling pathway-related protein p-AKT/AKT in the MCF-7, BT-549, MDA-MB-231, T47D, and MCF-10A cell lines was detected with western blot (Fig. 1A). The results showed that p-AKT (Thr308) and p-AKT (Ser473) were most significantly expressed in the MCF-7 cell line compared with MCF-10A ( $<$ < 0.05). Therefore, the MCF-7 cell line was used for the subsequent experiments.

\section{Effects of different concentrations of PTX on the viability of MCF-7 cells}

The MCF-7 cells were treated with different concentrations of PTX for $48 \mathrm{~h}$, and cell viability was measured using MTT assay (Fig. 2A). The results showed that cell viability decreased significantly as the concentration of PTX increased $(\mathrm{p}<0.05)$. When the PTX concentration reached $1 \mu \mathrm{M}$, cell viability was $50 \%$. The LDH cytotoxicity assay showed that cell death increased significantly as the PTX concentration increased ( $\mathrm{p} 0.05)$. When the PTX concentration was $1 \mu \mathrm{M}$, cell mortality was about $40 \%$ (Fig. 2B).
A

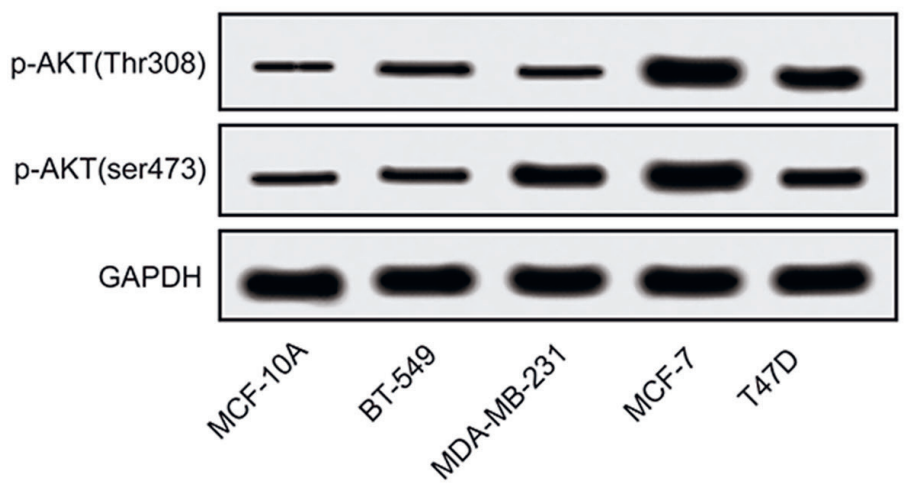

B
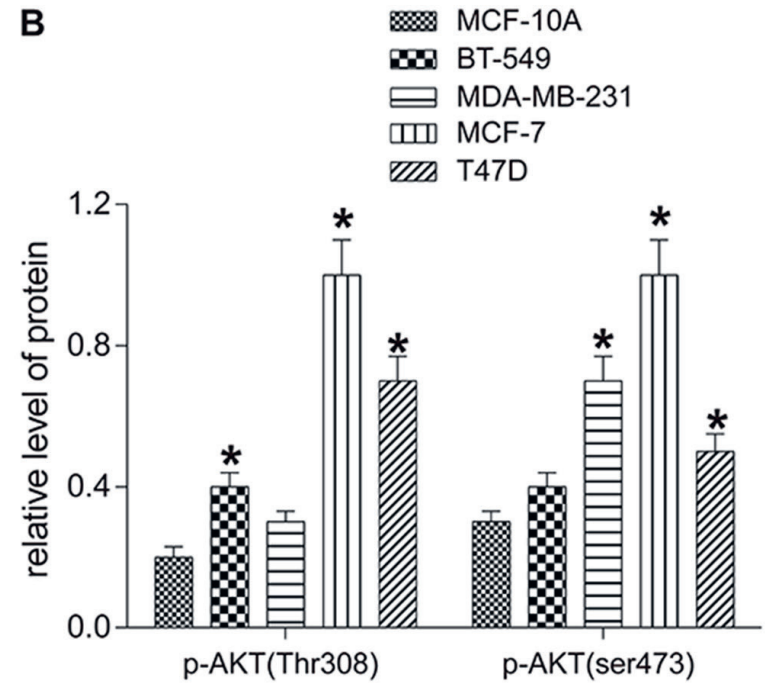

Fig. 1. Expression levels of the PI3K/AKT signaling pathway-related proteins in various cell lines. A. Expression of p-AKT (Thr308) and p-AKT (Ser473) detected with western blot in cell lines. B. Quantification of the western blot results 


\section{Effects of different concentrations of PTX on the proliferation of MCF-7}

Cell proliferation at different concentrations of PTX was measured using EdU assay (Fig. 3). The results showed that cell proliferation was inhibited by PTX in a dose-dependent manner. Cell proliferation was significantly decreased as the concertation of PTX increased ( $<<0.05)$. Therefore, PTX at the concentration of $1 \mu \mathrm{M}$ was used for the subsequent experiments.

\section{Comparison of cell apoptosis and cell cycle after PI3K overexpression and PTX treatment}

Based on the results outlined above, MCF-7 cells were selected and divided into 5 groups in the subsequent experiments: the control group (normal MCF-7 cells); the PTX group (treated with $1 \mu \mathrm{M}$ of PTX); the oe-PI3K group (transfected with PI3K overexpression plasmid
pcDNA3.1-PI3K); the NC-PI3K group (transfected with the negative control of pcDNA3.1-PI3K); and the oePI3K+PTX group (transfected with pcDNA3.1-PI3K and treated with $1 \mu \mathrm{M}$ of PTX).

Apoptosis and cell cycles were detected using flow cytometry (Fig. 4A,B). Compared with the control group, the NC-PI3K group had similar results, while the PTX group had a significantly higher cell apoptosis rate and a significantly higher number of cells in the G2/M phase $(\mathrm{p}<0.05)$. Compared with the PTX group, the cell apoptosis rate in the oe-PI3K group was significantly decreased, but the number of cells in the G2/M phase was increased $(\mathrm{p}<0.05)$. In the oe-PI3K+PTX group, the cell behaviors in the PTX group were partly reversed $(\mathrm{p}<0.05)$.

The expression levels of the apoptosis-related proteins $\mathrm{Bax}, \mathrm{Bcl}-2$ and cleaved caspase- 3 were detected using western blot in MCF-7 cells (Fig. 4C). Compared with the control group, the NC-PI3K group had similar results, while the expression levels of Bax and cleaved caspase-3 were upregulated in the PTX group; however, the expression
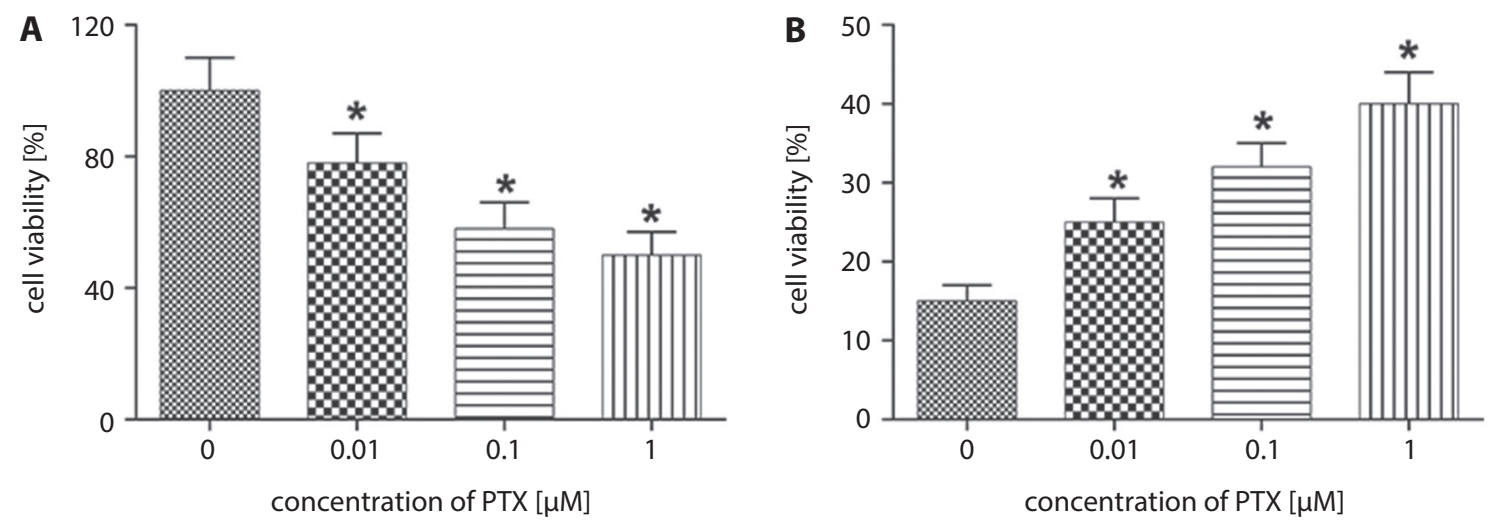

Fig. 2. Effects of different concentrations of PTX on the viability of MCF-7 cells. A. Cell viability detected with MTT assay. B. Cell death detected with LDH cytotoxicity assay

* $\mathrm{p}<0.05$, compared with when the PTX concentration is $0 \mu \mathrm{M}$.
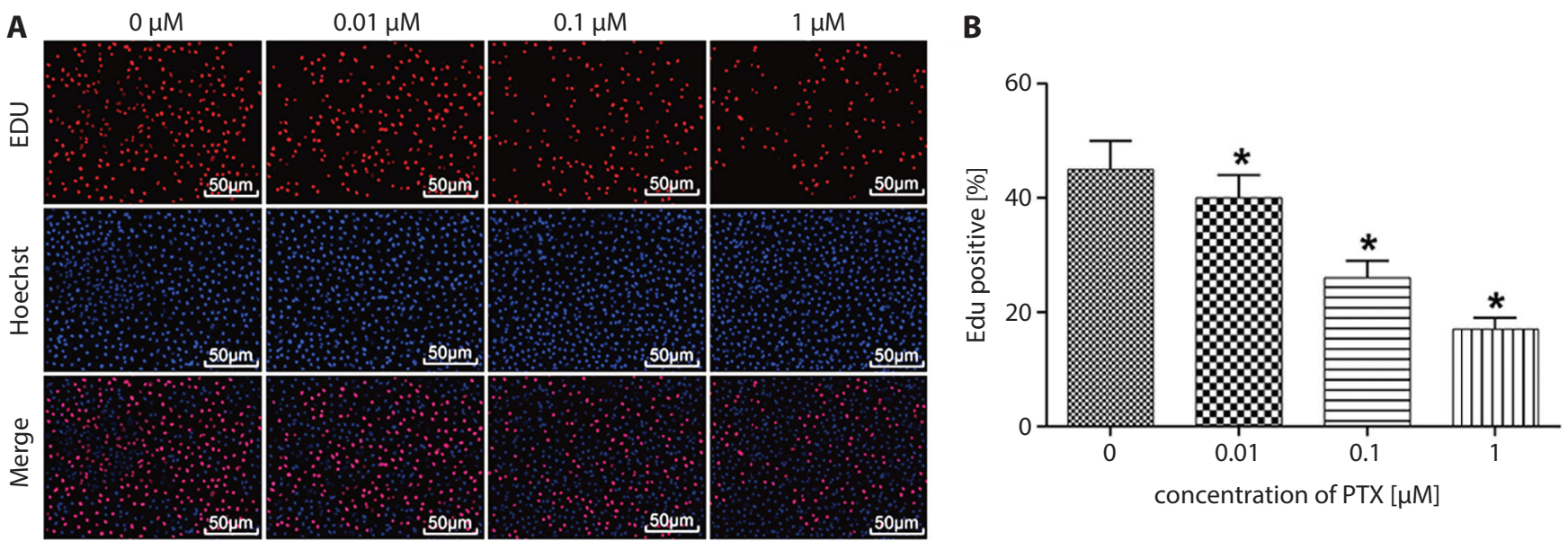

Fig. 3. Effects of different concentrations of PTX on the proliferation of MCF-7 cells. A. Cell proliferation detected with EdU proliferation assay. B. Percentage of EdU positive cells

${ }^{*} \mathrm{p}<0.05$, compared with cells without PTX treatment. 
A

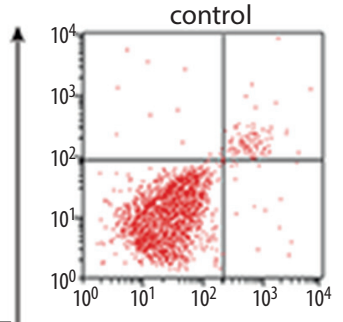

$\bar{\alpha}$

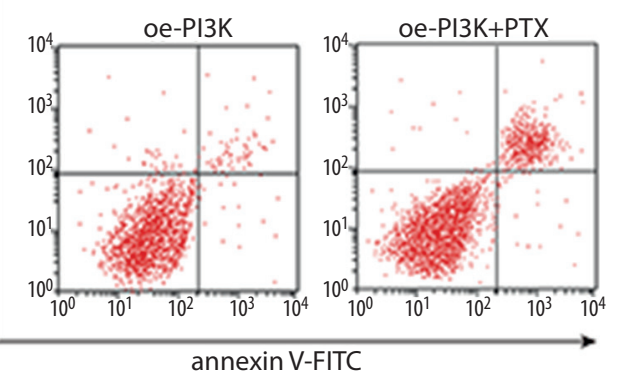

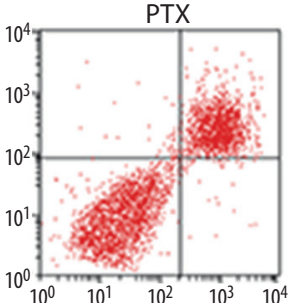
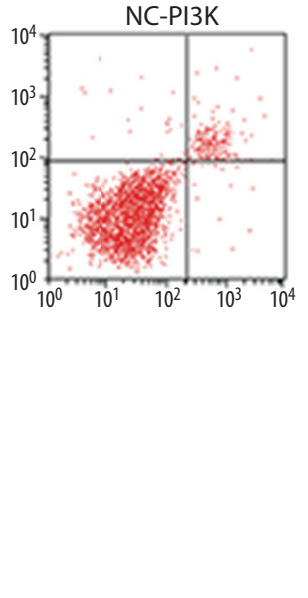
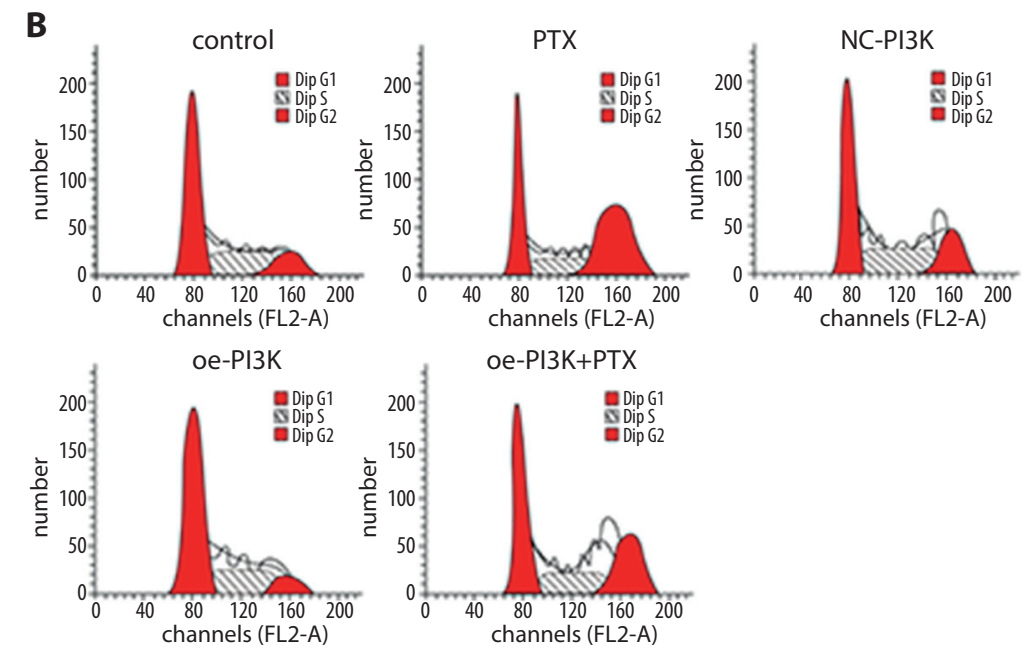

C
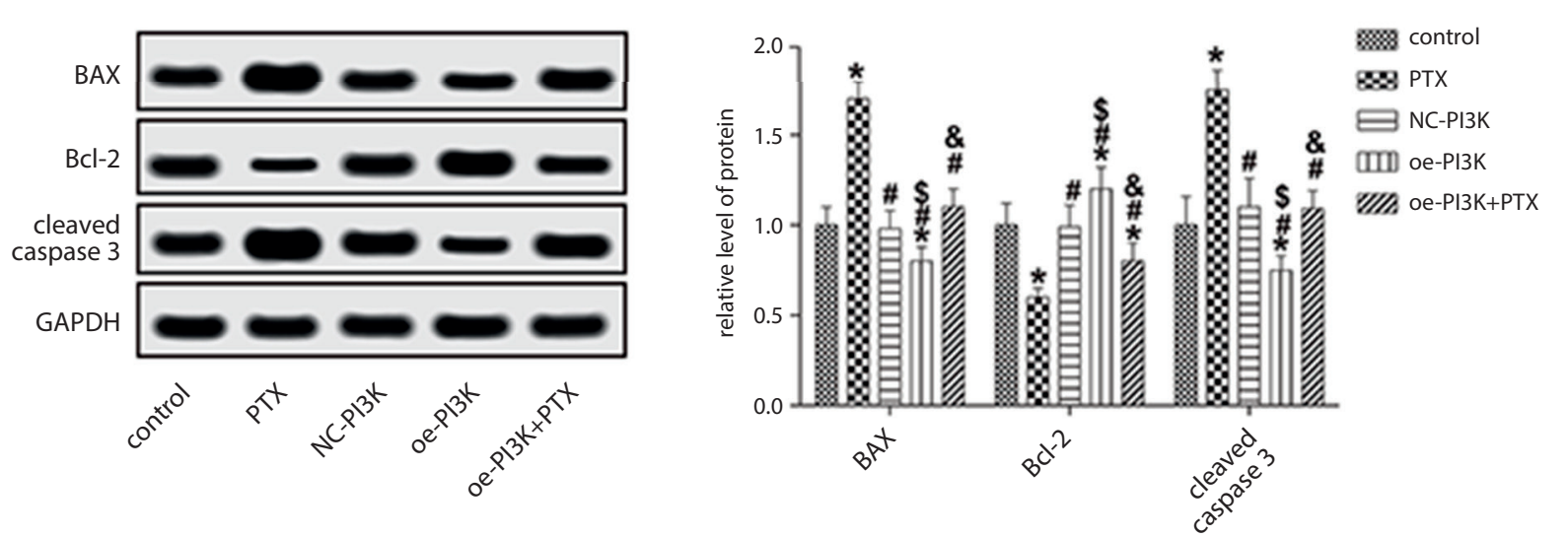

Fig. 4. Detection of apoptosis rate and cell-cycle distribution after PI3K overexpression and PTX treatment in MCF-7 cells. A. Cell apoptosis rate detected with flow cytometry. B. Cell-cycle distribution detected with flow cytometry. C. Expression level of apoptosis-related proteins detected with western blot

${ }^{*} p<0.05$ compared with the control group; ${ }^{*} p<0.05$ compared with the PTX group; ${ }^{\$} p<0.05$ compared with the NC-PI3K group; ${ }^{\&} p<0.05$ compared with the oe-PI3K group.

level of Bcl-2 was decreased in the PTX group $(\mathrm{p}<0.05)$. The expression levels of these proteins showed opposite trends in the oe-PI3K group when compared with the PTX group ( $\mathrm{p}<0.05)$. In the oe-PI3K+PTX group, the expression levels of related proteins in the PTX group was partly reversed (all $\mathrm{p}<0.05$ ).

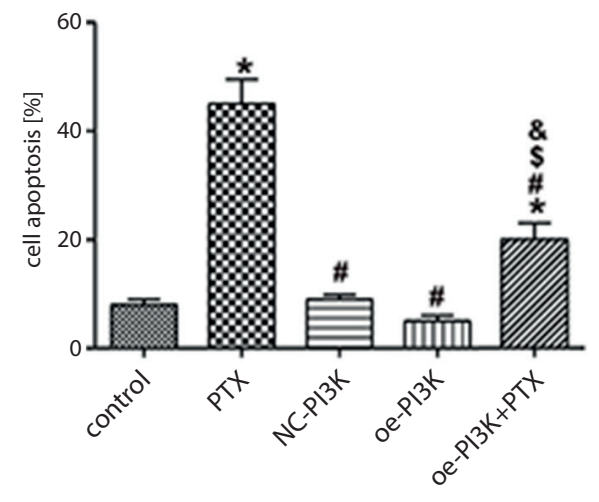

control

$\mathbf{B O 2}$ PTX

$\rightleftarrows$ NC-PI3K III oe-PI3K

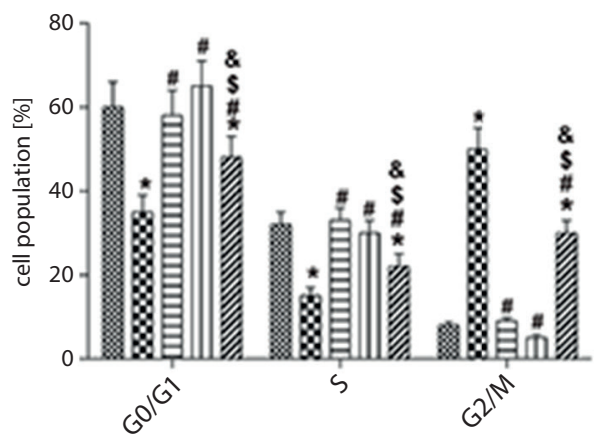


A
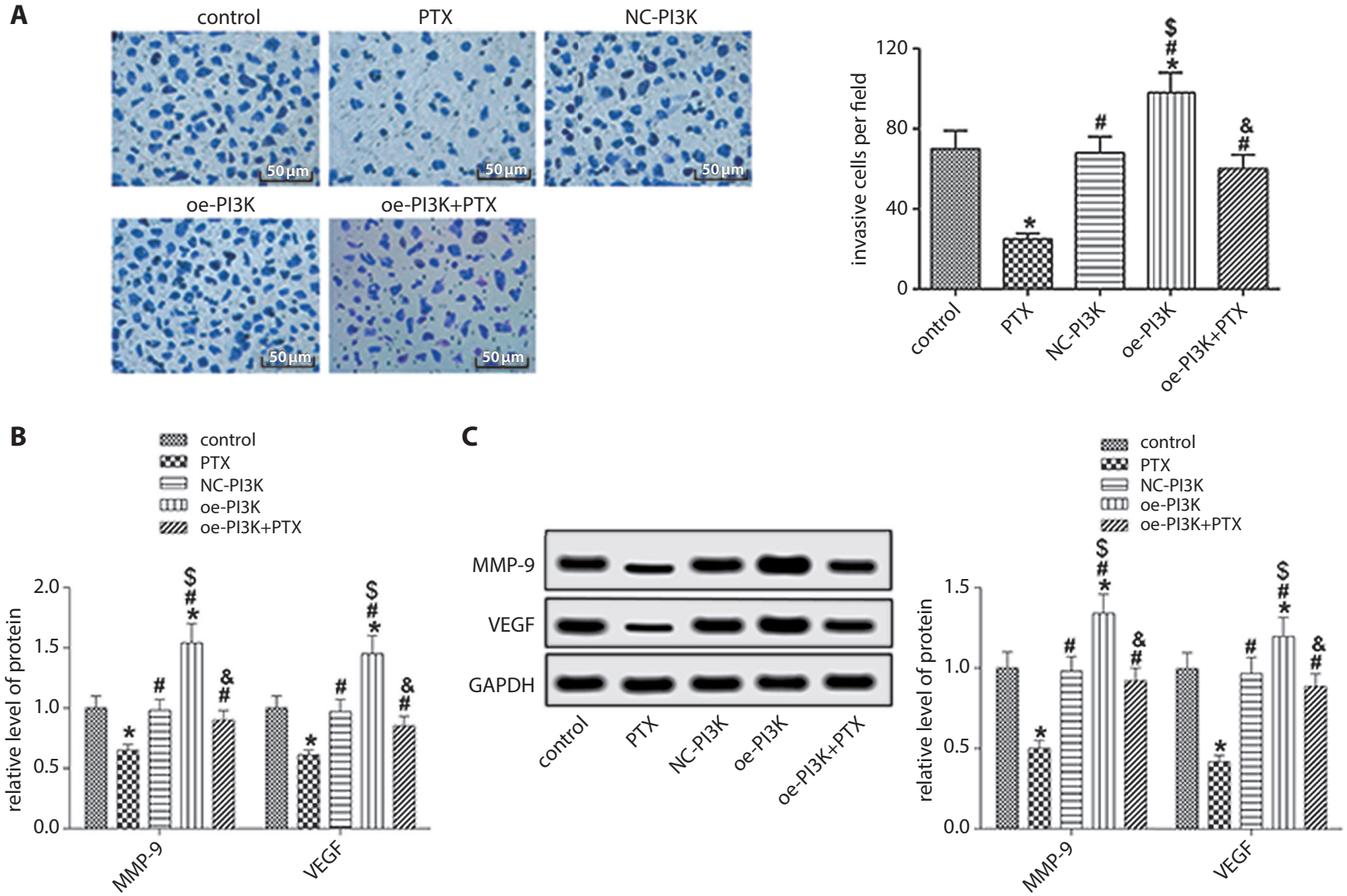

Fig. 5. Invasion assay after PI3K overexpression and PTX treatment in MCF-7 cells. A. Transwell invasion assay and transmembrane cell count. B. mRNA expression level of MMP-9 and VEGF detected with qRT-PCR. C. Protein expression level of MMP-9 and VEGF detected with western blot

${ }^{*} p<0.05$ compared with the control group; ${ }^{*} p<0.05$ compared with the PTX group; $\$ p<0.05$ compared with the NC-PI3K group; ${ }^{\&} p<0.05$ compared with the oe-PI3K group.

protein expression levels of cell invasion-related factors MMP-9 and VEGF (Fig. 5B).

The control group and the NC-PI3K group had similar results, while the PTX group had significantly decreased cell invasion ability and lower MMP-9 and VEGF expression levels $(\mathrm{p}<0.05)$. However, the oe-PI3K group had significantly increased cell invasion ability and higher MMP-9 and VEGF expression levels when compared with the PTX group $(\mathrm{p}<0.05)$. In the oe-PI3K+PTX group, the cell behaviors and the expression levels of related proteins in the PTX group were partly reversed (all p $<0.05$ ).

\section{Paclitaxel inhibited activation of the PI3K/AKT signaling pathway in MCF-7 breast cancer cells}

Western blot was used to detect the expression of PI3K/AKT signaling pathway-related protein p-AKT in each group (Fig. 6). Compared with the control group, the expression levels of p-AKT (Thr308) and p-AKT (Ser473) were significantly downregulated in the PTX group, but significantly upregulated in the oe-PI3K group (all p < 0.05). Compared with the PTX group, the expression of p-AKT (Thr308) and p-AKT (Ser473) was significantly upregulated in the oe-PI3K+PTX group (both $\mathrm{p}<0.05)$. Compared with the oe-PI3K group, the $\mathrm{p}-\mathrm{AKT}$ (Thr308) and p-AKT (Ser473) expression levels were significantly downregulated in the oe-PI3K+PTX group (both $\mathrm{p}<0.05)$.

\section{Discussion}

Currently, chemotherapy is the mainstream treatment for breast cancer, and PTX has been widely used as a firstline treatment. ${ }^{21}$ This study confirmed the in vitro therapeutic effect of PTX on breast cancer. At the same time, we conducted a preliminary exploration of the underlying mechanism, and found that PTX can inhibit the proliferation of breast cancer cells by inhibiting the activation of the PI3K/AKT pathway.

Our study found that PTX could decrease the activity of breast cancer cells and inhibit proliferation in a dosedependent manner. Paclitaxel has been shown to inhibit the development of a variety of cancer cells, including oral squamous cell carcinoma and breast cancer cells. ${ }^{22,23}$ Meanwhile, we also found that PTX can induce cell-cycle arrest and promote apoptosis by regulating the expression level 
A

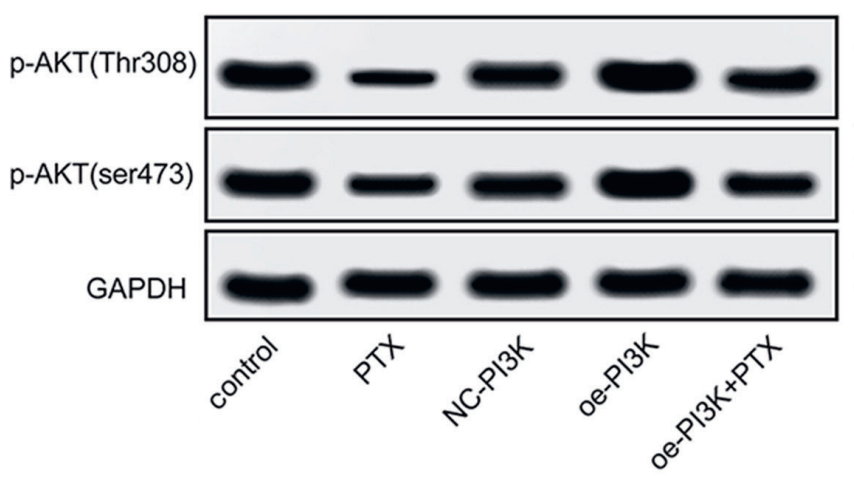

B

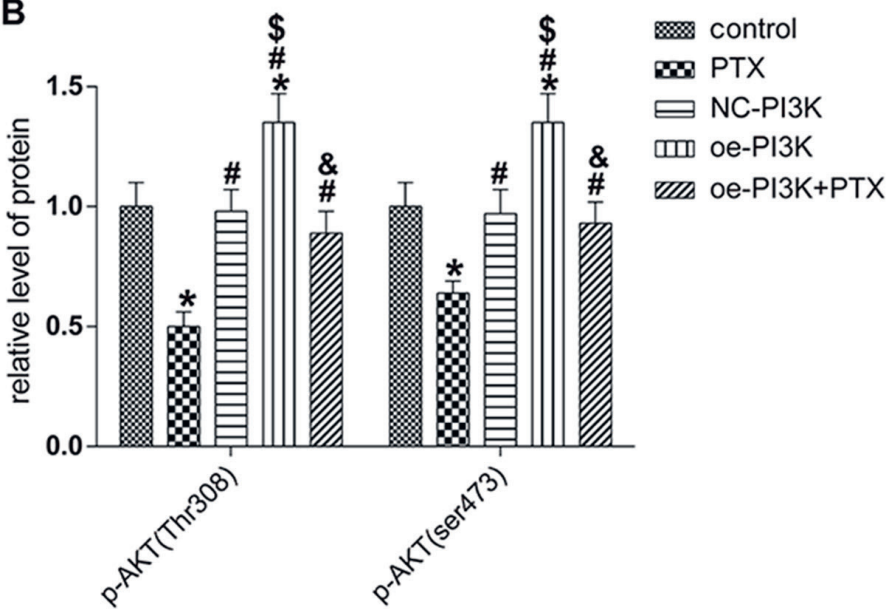

Fig. 6. Expression of p-AKT (Thr308) and p-AKT (Ser473) after PI3K overexpression and PTX treatment in MCF-7 cells. A. Expression level of p-AKT (Thr308) and P-AKT (Ser473) detected with western blot. B. Quantification result of western blot

${ }^{*} \mathrm{p}<0.05$ compared with the control group; ${ }^{*} \mathrm{p}<0.05$ compared with the PTX group; ${ }^{\mathrm{p}}<0.05$ compared with the NC-PI3K group; \& $<0.05$ compared with the oe-PI3K group.

of Bax, cleaved caspase-3 and Bcl-2. Kumari et al. discovered that coralyne and PTX have a synergistic effect on enhancing apoptosis in MDA-MB-231 cell line by arresting cells in the G1/S phase, inducing DNA fragmentation and changing the mitochondria membrane potential. ${ }^{24} \mathrm{Bax}$, cleaved caspase-3 and Bcl-2 are all apoptosis-related proteins that have a great impact on the development of many diseases. caspase- 3 and Bax are common pro-apoptotic proteins, while $\mathrm{Bcl}-2$ is an anti-apoptotic protein ${ }^{25,26}$; $\mathrm{Bcl}-2$ regulates mitochondrial membrane permeability, while Bax destroys mitochondrial outer membranes to promote apoptosis. ${ }^{27,28}$ The results of our study are consistent with the studies mentioned above, indicating that PTX could exert its therapeutic effect by inhibiting the proliferation of breast cancer cells and promoting their apoptosis.

In addition, PTX could also reduce the invasive ability of breast cancer cells, and the expression of invasion-related proteins MMP-9 and VEGF was inhibited in a dosedependent manner. Ismail et al. also found that PTX inhibited breast cancer cell invasion through the DJ-1/ KLF17 signaling pathway. ${ }^{29}$ The VEGF is an angiogenic protein that strongly promotes mitosis of vascular endothelial cells and is an important angiogenic cytokine. Miller et al. confirmed that VEGF was an important factor in the development and progression of breast cancer, and its overexpression could increase the resistance of breast cancer cells to PTX. ${ }^{30}$ The MMP-9 is a zinccontaining enzyme that participates in the regulation of the tumor microenvironment. ${ }^{31}$ Jiang et al. reported that PTX reduced MMP-9 expression and inhibited cell proliferation in ovarian cancer cells. ${ }^{32}$ The results of our study are consistent with these studies. Therefore, we confirmed the inhibitory effect of PTX on the invasion of breast cancer cells.
To further investigate the mechanism of PTX in the treatment of breast cancer, we examined the expression of p-AKT, which is a member of the PI3K/AKT signaling pathway. The PI3K/AKT signaling pathway has been shown to be critical for cell growth and survival. ${ }^{33}$ Activation of the PI3K/AKT pathway could lead to increased proliferation and metastasis of various malignancies, including non-small cell lung cancer, ovarian cancer and gastric cancer. ${ }^{34-36}$ Similarly, in breast cancer, inhibition of the PI3K/AKT signaling pathway could decrease cell proliferation and resistance to chemotherapeutic drugs. ${ }^{37,38}$ We found that the p-AKT (Thr308) and p-AKT (Ser473) expression levels in breast cancer cells were significantly reduced after PTX treatment. Moreover, after PI3K overexpression, the inhibitory effect of PTX on the growth of breast cancer cells was partly reversed. All these findings suggested that PTX could inhibit the proliferation and development of breast cancer by inhibiting the activity of the PI3K/AKT signaling pathway, which is the fundamental mechanism of its anti-cancer effect.

\section{Conclusions}

This study validated the potent efficacy of PTX as an anticancer chemotherapeutic drug in the treatment of breast cancer, and further found that it inhibited the development of malignancies by inhibiting the activation of the PI3K/AKT signaling pathway. Therefore, PTX combined with other drugs that can target the PI3K/AKT signaling pathway may further enhance its efficacy in the treatment of breast cancer. However, these results still need to be confirmed by further clinical trials, which we are planning to carry out in the future. 


\section{ORCID iDs}

Gang Li (1) https://orcid.org/0000-0002-1300-2449

Dongxin Xu (1) https://orcid.org/0000-0002-8728-0898

Jinju Sun (1) https://orcid.org/0000-0002-4277-5686

Shiyun Zhao (1) https://orcid.org/0000-0001-6883-6646

Dan Zheng (D) https://orcid.org/0000-0002-4206-8623

\section{References}

1. Shamsi M, Pirayesh Islamian J. Breast cancer: Early diagnosis and effective treatment by drug delivery tracing. Nucl Med Rev Centr East Eur. 2017;20(1):45-48.

2. Zhang Y, Li H, Zhang J, et al. The combinatory effects of natural prod ucts and chemotherapy drugs and their mechanisms in breast cancer treatment. Phytochem Rev. 2019;19(6):1179-1197.

3. Shawky MS, Huo CW, Henderson MA, Redfern A, Britt K, Thompson EW A review of the influence of mammographic density on breast cancer clinical and pathological phenotype. Breast Cancer Res Treat. 2019; 177(2):251-276.

4. Miles RC, Lehman CD, Mercaldo SF, Tamimi RM, Dontchos BN, Narayan AK. Obesity and breast cancer screening: Cross-sectional survey results from the behavioral risk factor surveillance system. Cancer. 2019; 125(23):4158-4163.

5. Jones ME, Schoemaker MJ, Wright LB, Ashworth A, Swerdlow AJ. Smoking and risk of breast cancer in the Generations Study cohort. Breast Cancer Res. 2017;19(1):118.

6. Wong G, Au E, Badve SV, Lim WH. Breast cancer and transplantation Am J Transplant. 2017;17(9):2243-2253.

7. Arpino G, Milano M, De Placido S. Features of aggressive breast cancer Breast. 2015;24(5):594-600.

8. Jia $Y$, Weng $Z$, Wang $C$, et al. Increased chemosensitivity and radio sensitivity of human breast cancer cell lines treated with novel functionalized single-walled carbon nanotubes. Oncol Lett. 2017;13(1): 206-214.

9. Lue X, Deng Q, Li H, Suo Z. Altered characteristics of cancer stem/ initiating cells in a breast cancer cell line treated with persistent 5-FU chemotherapy. Exp Ther Med. 2011;2(5):821-826.

10. Kantor O, Bao J, Jaskowiak N, Yao K, Tseng J. The prognostic value of the AJCC $8^{\text {th }}$ Edition Staging System for patients undergoing neoadjuvant chemotherapy for breast cancer. Ann Surg Oncol. 2020;27(2): 352-358.

11. Bressand D, Novell A, Girault A, et al. Enhancing Nab-paclitaxel delivery using microbubble-assisted ultrasound in a pancreatic cancer model. Mol Pharm. 2019;16(9):3814-3822.

12. Leung JC, Cassimeris L. Reorganization of paclitaxel-stabilized microtubule arrays at mitotic entry: Roles of depolymerizing kinesins and severing proteins. Cancer Biol Ther. 2019;20(10):1337-1347.

13. Ma X, Özliseli E, Zhang Y, Pan G, Wang D, Zhang H. Fabrication of redox-responsive doxorubicin and paclitaxel prodrug nanoparticles with microfluidics for selective cancer therapy. Biomater Sci. 2019;7(2):634-644.

14. Fukuizumi A, Minegishi Y, Omori M, et al. Weekly paclitaxel in combination with carboplatin for advanced non-small-cell lung cancer complicated by idiopathic interstitial pneumonias: A single-arm phase II study. Int J Clin Oncol. 2019;24(12):1543-1548.

15. Zou L, Wang D, Hu Y, et al. Drug resistance reversal in ovarian cancer cells of paclitaxel and borneol combination therapy mediated by PEG-PAMAM nanoparticles. Oncotarget. 2017;8(36):60453-60468.

16. Hoch MA, Cousins K, Nartey R, Riley K, Hartranft M. Two cases of combination therapy with cetuximab, paclitaxel, and cisplatin for advanced head and neck cancer. J Oncol Pharm Pract. 2018;24(7):553-554.

17. Xie HJ, Zhao J, Zhuo-Ma D, Zhan-Dui N, Er-Bu A, Tsering T. Inhibiting tumour metastasis by DQA modified paclitaxel plus ligustrazine micelles in treatment of non-small-cell lung cancer. ArtifCells Nanomed Biotechnol. 2019;47(1):3465-3477.

18. Takashima T, Kawajiri H, Nishimori T, et al. Safety and efficacy of lowdose nanoparticle albumin-bound paclitaxel for HER2-negative metastatic breast cancer. Anticancer Res. 2018;38(1):379-383.
19. Mendez-Pertuz M, Martinez P, Blanco-Aparicio C, et al. Modulation of telomere protection by the PI3K/AKT pathway. Nat Commun. 2017; 8(1):1278.

20. Qu Y, Hao C, Xu J, et al. ILK promotes cell proliferation in breast cancer cells by activating the PI3K/Akt pathway. MolMed Rep. 2017;16(4): 5036-5042.

21. Zheng $P$, Dong $L$, Zhang $B$, et al. Long noncoding RNA CASC2 promotes paclitaxel resistance in breast cancer through regulation of miR-18a-5p/CDK19. Histochem Cell Biol. 2019;152(4):281-291.

22. Hu J, Zhang N, Wang R, Huang F, Li G. Paclitaxel induces apoptosis and reduces proliferation by targeting epidermal growth factor receptor signaling pathway in oral cavity squamous cell carcinoma. Oncol Lett. 2015;10(4):2378-2384.

23. Liu M, Gong C, Xu R, et al. MicroRNA-5195-3p enhances the chemosensitivity of triple-negative breast cancer to paclitaxel by downregulating EIF4A2. Cell Mol Biol Lett. 2019;24:47.

24. Kumari S, Mohan MG, Shailender G, et al. Synergistic enhancement of apoptosis by coralyne and paclitaxel in combination on MDAMB-231, a triple-negative breast cancer cell line. J Cell Biochem. 2019; 120(10):18104-18116

25. Freitas M, Alves V, Sarmento-Ribeiro AB, Mota-Pinto A. Combined effect of sodium selenite and docetaxel on PC3 metastatic prostate cancer cell line. Biochem Biophys Res Commun. 2011;408(4):713-719.

26. Xiang $X Y$, Kang JS, Yang XC, et al. SIRT3 participates in glucose metabolism interruption and apoptosis induced by $\mathrm{BH} 3$ mimetic $\mathrm{Si}$ in ovarian cancer cells. Int J Oncol. 2016;49(2):773-784.

27. Lv J, Liang Y, Tu Y, Chen J, Xie Y. Hypoxic preconditioning reduces propofol-induced neuroapoptosis via regulation of $\mathrm{BCl}-2$ and $\mathrm{Bax}$ and downregulation of activated caspase-3 in the hippocampus of neonatal rats. Neurol Res. 2018;40(9):767-773.

28. Peng X, Chen K, Chen J, et al. Aflatoxin B1 affects apoptosis and expression of $\mathrm{Bax}, \mathrm{Bcl}-2$, and caspase- 3 in thymus and bursa of fabricius in broiler chickens. Environ Toxicol. 2016;31(9):1113-1120.

29. Ismail IA, Ei-Sokkary GH, Saber SH. Low doses of paclitaxel repress breast cancer invasion through DJ-1/KLF17 signaling pathway. Clin Exp Pharmacol Physiol. 2018;45:961-968.

30. Miller K, Wang M, Gralow J, et al. Paclitaxel plus bevacizumab versus paclitaxel alone for metastatic breast cancer. N Engl J Med. 2007; 357(26):2666-2676.

31. Kerenidi T, Kazakou AP, Lada M, et al. Clinical significance of circulating osteopontin levels in patients with lung cancer and correlation with VEGF and MMP-9. Cancer Invest. 2016;34(8):385-392.

32. Jiang Z, Zhang SJ, Chen B, et al. Paclitaxel inhibited proliferation and matrix metalloproteinases (MMP-2, MMP-9) expression in ovarian cancer HO8910 cells. Chinese Journal of Pharmaceutical Analysis. 2002;22:458-460.

33. Ren $F$, Su H, Jiang $H$, Chen Y. Overexpression of miR-623 suppresses progression of hepatocellular carcinoma via regulating the $\mathrm{PI} 3 \mathrm{~K} / \mathrm{Akt}$ signaling pathway by targeting XRCC5. J Cell Biochem. 2020;121(34): 213-223.

34. Zhu X, Jiang H, Li J, Xu J, Fei Z. Anticancer effects of Paris saponins by apoptosis and PI3K/AKT pathway in gefitinib-resistant non-small cell lung cancer. Med Sci Monit. 2016;22:1435-1441.

35. Meng Q, Xia C, Fang J, Rojanasakul Y, Jiang B-H. Role of PI3K and AKT specific isoforms in ovarian cancer cell migration, invasion and proliferation through the p70S6K1 pathway. Cell Sign. 2006;18(12): 2262-2271.

36. Sun C, Tao Y, Gao Y, et al. F-box protein 11 promotes the growth and metastasis of gastric cancer via PI3K/AKT pathway-mediated EMT. Biomed Pharmacother. 2018;98:416-423.

37. Liu T, Guo J, Zhang X. MiR-202-5p/PTEN mediates doxorubicin-resistance of breast cancer cells via PI3K/Akt signaling pathway. Cancer Biol Ther. 2019;20(7):989-998.

38. Li N, Miao Y, Shan Y, et al. MiR-106b and miR-93 regulate cell progression by suppression of PTEN via PI3K/Akt pathway in breast cancer. Cell Death Dis. 2017;8(5):e2796 